\title{
DENSENESS, MAXIMALITY, AND DECIDABILITY OF GRAMMATICAL FAMILIES ${ }^{1)}$
}

\author{
H. A. MAURER, A. SAlOMAA, E. WELZL and D. WOOD
}

We demonstrate that there is no sub-regular maximally dense interval of grammatical families by way of two characterizations of sub-regular dense intervals. Moreover we prove that it is decidable whether or not a given sub-regular interval is dense. These results are proved using the twin notions of language forms and linguistical families that are of interest in their own right.

\section{Introduction and overview}

The study of grammatical similarity via the tool of grammar forms now forms a substantial chapter in the development of formal language theory. Not only has grammar form theory contributed to our understanding of similarity, but it has also raised many challenging and interesting problems. It is the purpose of this paper to present the solution to one of these problems. The problem we tackle is found when trying to refine some basic hierarchy results for language families. To explore this further we need to first introduce grammar forms and their related language families. A (context-free) grammar form is simply a context-free grammar $G=(V, \Sigma, P, S)$, where, as usual, $V$ is a finite alphabet, $\Sigma \subseteq V$ is a terminal alphabet and $V-\Sigma$ is the nonterminal alphabet, $P \subseteq(V-\Sigma) \times V^{*}$ is a finite set of productions, where a production $(A, \alpha)$ is usually written as $A \rightarrow \alpha$, and $S$ in $V-\Sigma$ is a sentence symbol. We use $L(G)$ to denote the language generated by $G$, as usual.

Given two grammars $G^{\prime}=\left(V^{\prime}, \Sigma^{\prime}, P^{\prime}, S^{\prime}\right)$ and $G=(V, \Sigma, P, S)$ we say $G^{\prime}$ is an interpretation of $G$, denoted by $G^{\prime} \leqq G$ if there is a (strict alphabetic) morphism $h: V^{\prime} \rightarrow V$ such that $h\left(V^{\prime}-\Sigma^{\prime}\right) \subseteq V-\Sigma, h\left(\Sigma^{\prime}\right) \subseteq \Sigma, h\left(P^{\prime}\right) \subseteq P, \quad h\left(S^{\prime}\right)=S$, where $h\left(P^{\prime}\right)=\left\{h(A) \rightarrow h(\alpha): A \rightarrow \alpha\right.$ is in $\left.P^{\prime}\right\}$. A morphism is strict-alphabetic if it maps letters to letters; all morphisms considered in this paper are strict alphabetic. Associated with each grammar $G$ under interpretation is a family of languages called the grammatical family of $G$. It is denoted by $L(G)$ and is defined as

1) Work carried out under the auspices of the Natural Sciences and Engineering Research Council of Canada Grant No. A-5192. 
$L(G)=\left\{L\left(G^{\prime}\right): G^{\prime} \leqq G\right\}$. When a grammar is interpreted in this way it is often called a grammar form. Since the relation $\leqq$ is reflexive and transitive $L\left(G^{\prime}\right) \subseteq \boldsymbol{L}(G)$ whenever $G^{\prime} \leqq G$. Thus it is natural to consider the partially-ordered set of all grammatical families ordered with respect to containment. Such investigations are traditional in formal language theory, leading to numerous hierarchy results.

For $i \geqq 1$, let $F_{i}$ be $S \rightarrow a^{j}, 1 \leqq j \leqq i$. Then $L\left(F_{i}\right)$ is finite as is $L\left(F_{i}^{\prime}\right)$, for all $F_{i}^{\prime} \leqq F_{i}$. Moreover $L\left(F_{i}\right) \subseteq L\left(F_{i+1}\right)$. It is not difficult to show that

$$
\boldsymbol{L}\left(F_{1}\right) \subset \boldsymbol{L}\left(F_{2}\right) \subset \boldsymbol{L}\left(F_{3}\right) \subset \ldots \subset \boldsymbol{L}(R E G) .
$$

In a similar manner, based on deeper results in the theory it is possible to demonstrate infinite hierarchies of regular families, linear families, and context-free families. Showing the existence of such hierarchies, which are paths in the poset of grammatical families, is only a first step in obtaining a better understanding of the structure of this poset. It should be noted that the coarser interpretation relation, the first one to be introduced and studied by [CG] leads to a much simpler poset structure as the recent papers [GGS1] and [GGS2] demonstrate. In our setting a reasonable question is: whenever $L\left(G_{1}\right) \subset \boldsymbol{L}\left(G_{2}\right)$ for two grammars $G_{1}$ and $G_{2}$ does there exist $G_{3}$ with $L\left(G_{1}\right) \subset L\left(G_{3}\right) \subset L\left(G_{2}\right)$ ? That such is not always the case is seen by considering the following pair of grammars:

$$
G_{1}: S \rightarrow a b \quad G_{2}: S \rightarrow a b \mid c d e .
$$

Clearly $L\left(G_{1}\right) \subset \boldsymbol{L}\left(G_{2}\right)$ by the obvious length argument. That there is no $G_{3}$ properly in between is demonstrated as follows.

First observe that for finite forms $G$ and $H$ with $S$ as their only nonterminal $\boldsymbol{L}(G) \subset \boldsymbol{L}(H)$ if and only if $G \leqq H$ and $H$, where means 'is not an interpretation of', that is $G<H$. Clearly $G \leqq H$ implies $\boldsymbol{L}(G) \leqq \boldsymbol{L}(H)$. However if $\boldsymbol{L}(G) \subseteq$ $\boldsymbol{L}(H)$ then $L(G)$ is in $\boldsymbol{L}(H)$ and hence there is a grammar $F \leqq H$ with $L(F)=$ $L(G)$. But $G$ and $H$ have the same simple form therefore $G \leqq F$ and, hence, $G \leqq H$. Finally proper inclusion implies $H \neq G$ by a similar argument.

Other examples of this kind are easily obtained, however what happens when there is no difference in the lengths of words generated by the two grammars? For example let $G_{1}: S \rightarrow a b ; G_{2}: S \rightarrow a a$ then $L\left(G_{1}\right) \subset L\left(G_{2}\right)$ and all words are of length two. In [MSW1] this led to the notion of interpretations of directed graphs and hence to directed graph families, see [S]. Basically each word specifies an edge, so $a b$ is an edge between nodes $a$ and $b$. It was demonstrated in [MSW1] that there are infinitely many grammatical families between $L\left(G_{1}\right)$ and $L\left(G_{2}\right)$. Moreover for any two families $G_{3}$ and $G_{4}$ satisfying $L\left(G_{1}\right) \subseteq L\left(G_{3}\right) \subset L\left(G_{4}\right) \leqq L\left(G_{2}\right)$ there is a $G_{5}$ properly in between $G_{3}$ and $G_{4}$, that is $L\left(G_{3}\right) \subset L\left(G_{5}\right) \subset L\left(G_{4}\right)$. For this reason we say that the interval defined by $L\left(G_{1}\right)$ and $L\left(G_{2}\right)$, denoted by $\left(G_{1}, G_{2}\right)$, is dense. In [MSW3] a quite surprising result is proved, namely, the interval $\left(G^{\prime}, G\right)$ is dense, whenever $L\left(G^{\prime}\right)=L(R E G)$ and $L(G)=L(C F)$. Thus there are dense intervals of sub-regular grammatical families and also dense intervals of super-regular grammati- 
cal families. One basic question about such intervals is: Are there maximal dense intervals? That is are there dense intervals which cannot be extended either above or below while retaining density. In this paper we partially solve this problem for regular intervals by demonstrating that there are no maximal dense regular intervals whose upper family is $\boldsymbol{L}_{R E G}$. Extending this result to all regular dense intervals is not immediate, even if it holds, whereas for context-free dense intervals it probably does not hold.

Apart from this partial solution to the maximality question we also demonstrate that denseness is decidable for regular intervals. It has recently been shown that denseness is undecidable for context-free intervals [N].

These solutions are obtained by way of language forms and linguistical families, concepts introduced in [MSW4] and further investigated in [MSW5]. For a regular grammar form $G$ is it well-known [OSW] that $L(G)$ is characterized completely by $L(G)$, in the following sense. Consider a regular language $L^{\prime} \subseteq \Sigma^{*}$ and let $L=L(G)$ with $\Sigma$ the alphabet of $L$. We write $L^{\prime} \leqq L$ if there is a strict alphabetic morphism $h: \Sigma^{* *} \rightarrow \Sigma^{*}$ such that $h\left(L^{\prime}\right) \subseteq L$. In analogy with the introduction of the grammatical family of a grammar form we define the regular linguistical family of the regular language form $L$ by: $L_{r}(L)=\left\{L^{\prime}: L^{\prime} \leqq L\right.$ and $L^{\prime}$ is regular $\}$. It is proved in [OSW] that if $L(G) \subseteq L(R E G)$ then $L(G)=L_{r}(L(G))$. This characterization implies that we need only treat regular language forms and regular linguistical families, rather than the more indirect (regular) grammar forms and regular grammatical families.

\section{Some definitional and theoretical preliminaries}

Given a language $L$ and a language $L^{\prime}$ we say $L^{\prime}$ is an interpretation of $L$ if there is a strict alphabetic morphism $h$ such that $h\left(L^{\prime}\right) \subseteq L$. We denote this by $L^{\prime} \leqq L$. We say $L^{\prime}$ is a regular interpretation of $L$ if $L^{\prime} \leqq L$ and $L^{\prime}$ is regular, this is denoted by $L^{\prime} \leqq_{r} L$. Note that $L$ itself need not be regular. Similarly we say $L^{\prime}$ is a finite interpretation of $L$, denoted by $L^{\prime} \leqq{ }_{f} L$, if $L^{\prime} \leqq L$ and $L^{\prime}$ is finite. Moreover, we write $L^{\prime}<L\left(L^{\prime}<_{r} L, L^{\prime}<_{f} L\right)$ if $L^{\prime} \leqq L$ but $L$ is not an interpretation of $L^{\prime}$ (and $L^{\prime}$ is regular, finite, respectively). If $L^{\prime} \leqq L$ and $L \leqq L^{\prime}$ then we say that $L$ and $L^{\prime}$ are equivalent, denoted by $L \sim L^{\prime}$.

The corresponding linguistical families are denoted by $\boldsymbol{L}(L), \boldsymbol{L}_{\boldsymbol{r}}(L)$, and $\boldsymbol{L}_{f}(L)$, respectively. These notions are tied together in the following theorem, see [MSW4].

The orem 2.1. For all languages $L_{1}$ and $L_{2}$ the following statements are equivalent:

(1) $L\left(L_{1}\right)=L\left(L_{2}\right)$

(2) $L_{r}\left(L_{1}\right)=L_{r}\left(L_{2}\right)$

(3) $L_{f}\left(L_{1}\right)=L_{f}\left(L_{2}\right)$.

The above theorem has the obvious implication that to obtain distinct linguistical families we only need obtain distinct regular-linguistical families or, even, distinct 
finite-linguistical families. These, it is assumed, will be easier to handle. Note that $\boldsymbol{L}\left(L_{1}\right) \subseteq \boldsymbol{L}\left(L_{2}\right)$ if and only if $L_{1} \leqq L_{2}$ if and only if $\boldsymbol{L}_{r}\left(L_{1}\right) \subseteq \boldsymbol{L}_{r}\left(L_{2}\right)$ if and only if $\boldsymbol{L}_{f}\left(L_{1}\right) \subseteq \boldsymbol{L}_{f}\left(L_{2}\right)$.

In analogy with the definition of dense interval for grammar forms we say that $\left(L_{1}, L_{2}\right)$ denotes an interval if $L_{1}<L_{2}$ and hence $\boldsymbol{L}\left(L_{1}\right) \subset \boldsymbol{L}\left(L_{2}\right)$. The interval $\left(L_{1}, L_{2}\right)$ is dense if for all languages $L_{3}$ and $L_{4}$ that satisfy $L_{1} \leqq L_{3}<L_{4} \leqq L_{2}$ there is an $L_{5}$ with $L_{3}<L_{5}<L_{4}$. Similarly we say that an interval $\left(L_{1}, L_{2}\right)$ is regular if both $L_{1}$ and $L_{2}$ are regular and it is regular dense, $r$-dense for short, if it is regular and for all regular languages $L_{3}$ and $L_{4}$ that satisfy $L_{1} \leqq_{r} L_{3}<_{r} L_{4} \leqq_{r} L_{2}$ there is a regular language $L_{5}$ with $L_{3}<_{r} L_{5}<_{r} L_{4}$.

We have defined these notions in terms of interpretations rather than in terms of linguistical families, but since $L_{1} \leqq L_{2}$ if and only if $L\left(L_{1}\right) \subseteq L\left(L_{2}\right)$ this is only a matter of convenience.

Density and regular density are somewhat related as we will show below, but we first need to define super-disjoint union.

Let $L_{1} \subseteq \Sigma_{1}^{*}$ and $L_{2} \subseteq \Sigma_{2}^{*}$ be two languages. Then the super-disjoint union of $L_{1}$ and $L_{2}$, denoted by $L_{1} \cup L_{2}$, is their union if $\Sigma_{1} \cap \Sigma_{2}=\emptyset$ and is undefined otherwise. We call it super-disjoint union since it is not only a disjoint union $\left(L_{1} \cap L_{2}=\emptyset\right)$, but also $\Sigma_{1} \cap \Sigma_{2}=\emptyset$. If $L_{1}$ and $L_{2}$ are arbitrary language forms, then we can always rename the alphabet of $L_{1}$, say, to obtain disjoint alphabets, hence, in this case, we assume that $L_{1} \cup L_{2}$ is always well-defined. We use the ordinary union sign for superdisjoint unions, because of typographical reasons.

We now relate dense and regular dense intervals.

Theorem 2.2. Let $\left(L_{1}, L_{2}\right)$ be a regular interval. If $\left(L_{1}, L_{2}\right)$ is dense then $\left(L_{1}, L_{2}\right)$ is regular dense.

Proof. Consider an arbitrary regular interval $\left(L_{3}, L_{4}\right)$ that satisfies $L_{1} \leqq_{r} L_{3}$ and $L_{4} \leqq_{r} L_{2}$; clearly such an interval always exists. Since $\left(L_{1}, L_{2}\right)$ is dense there is an $L_{5}$ with $L_{3}<_{r} L_{5}<L_{4}$. Now by Theorem 2.1 this implies there is a finite language $F$ which is an interpretation of $L_{5}$ but not of $L_{3}$. Consider $L=L_{3} \cup F$. Clearly $L_{3}<_{r} L$, $L$ is regular, therefore $L \leqq_{r} L_{5}$, and hence $L<_{r} L_{4}$. In other words $\left(L_{1}, L_{2}\right)$ is a regular dense interval.

If an interval $\left(L_{1}, L_{2}\right)$ contains no language $L$ properly in between $L_{1}$ and $L_{2}$, then we say that $L_{1}$ is a predecessor of $L_{2}$ and $L_{2}$ has a predecessor.

Predecessors and density are complementary notions, since we have:

Proposition 2.3. Let $\left(L_{1}, L_{2}\right)$ be an interval. Then $\left(L_{1}, L_{2}\right)$ is dense if and only if it contains no language $L$ having a predecessor in the interval.

It turns out that characterizing those languages which have predecessors is one step on the way to characterizing those intervals which are dense. For this purpose we require three auxiliary notions. 
Let $L$ be a language and $\Sigma$ be its alphabet. We say $L$ is coherent if for all nonempty disjoint alphabets $\Sigma_{1}$ and $\Sigma_{2}$ with $\Sigma_{1} \cup \Sigma_{2}=\Sigma$, there is a word $x$ in $L$ with $x$ in $\Sigma^{*} \Sigma_{1} \Sigma^{*} \Sigma_{2} \Sigma^{*} \cup \Sigma^{*} \Sigma_{2} \Sigma^{*} \Sigma_{1} \Sigma^{*}$. We say $L$ is incoherent otherwise. Observe that if $L$ is incoherent then there are $L_{1}$ and $L_{2}$ with $L=L_{1} \cup L_{2}$, where $\emptyset \neq L_{i} \neq\{\lambda\}, i=1,2$.

A language form $L$ is minimal if there is no language form $L^{\prime} \subset L$ with $L\left(L^{\prime}\right)=$ $\boldsymbol{L}(L)$. If $L$ is finite, then minimality is clearly decidable and if $L$ is finite and non-minimal then the construction of an equivalent minimal $L^{\prime} \subset L$ is straightforward.

We now introduce our third notion, looping languages. A language $L$ is looping if either $L$ contains a word containing two appearances of the same letter, or there exist distinct words $w_{1}, \ldots, w_{n}$ in $L$ and distinct letters $a_{1}, \ldots, a_{n}$ in $\operatorname{alph}(L)$, for $n \geqq 2$, such that $a_{i}$ and $a_{i+1}$ are in $w_{i}, 1 \leqq i<n$ and $a_{n}$ and $a_{1}$ are in $w_{n}$. If $L$ is not looping we say it is nonlooping. (alph $(L)$ is the smallest alphabet $\Sigma$ such that $L \subseteq \Sigma^{*}$.)

Given a language form $L, L^{\prime}$ is a nonlooping interpretation of $L$, denoted by $L^{\prime} \leqq_{n} L$ if $L^{\prime} \leqq L$ and $L^{\prime}$ is nonlooping. We therefore have $L_{n}(L)$ as well.

In [MSW2] the following result is to be found.

Proposition 2.4. Let $L$ be a finite language.

(i) If $L$ is minimal and coherent, then $L$ has a predecessor if and only if $L$ is nonlooping.

(ii) If $L$ is minimal, then $L$ has a predecessor if and only if $L=K \cup N$ for some $K$ and nontrivial $N$, where $N$ is nonlooping.

We extend this result to arbitrary languages, by first treating the coherent case.

Theorem 2.5. Let $L$ be a coherent minimal language. Then $L$ has a predecessor if and only if $L$ is nonlooping.

Proof. If $L$ is finite the result follows by Proposition 2.4, therefore assume $L$ is infinite. Since each language is over a finite alphabet an infinite language is always looping. Therefore we only need demonstrate that an infinite language never has a predecessor to complete the Theorem. Assume $L$ has a predecessor $P$. We argue by contradiction demonstrating that there is always a language properly in between $P$ and $L$. By Theorem 2.1, there exists a finite $F$ with $F \leqq L$ and $F$ 秉 $P$. This implies $P<P \cup F \leqq L$. We also have $L \neq P \cup F$. This follows from the coherence of $L$, the finiteness of $F$, and $P<L$. Thus $P<P \cup F<L$ and we have obtained a language properly in between $P$ and $L$ as required, therefore $L$ has no predecessor.

We now generalize the second part of Proposition 2.4.

Theorem 2.6. Let $L$ be a minimal language. Then $L$ has a predecessor if and only if $L=K \cup N$, for some language $K$ and some nontrivial, nonlooping $N$.

Proof. The proof for finite $L$ is to be found in [MSW2]. The infinite case follows analogously, we merely give a brief proof sketch. Assume $L$ is infinite. If $L$ is coherent then $L$ has no predecessor by Theorem 2.5 and it has no decomposition of the required 
form. Thus the Theorem holds in this case. Therefore assume $L$ is incoherent. If $L=$ $K \cup N$, where $N$ is nontrivial and nonlooping, then $N$ has a predecessor $P$ and we need to show that $K \cup P$ is a predecessor of $L$. On the other hand if $L$ has no nontrivial, nonlooping component $N$, then it only remains to demonstrate that there is a language properly in between $P$ and $L$ for any $P<L$. In both cases we make heavy use of the observation that if a coherent language $Q$ satisfies $Q \leqq L$, then $Q$ is an interpretation of some coherent component of $L$.

\section{The density characterization theorems}

One of the major obstacles to proving decidability results for intervals of grammatical families has been the lack of a density characterization theorem for such intervals. In the present section we provide such theorems which are then used to provide examples of dense intervals.

First we need to introduce some additional notation and terminology concerning nonlooping languages. We say that two languages $L_{1}$ and $L_{2}$ are nonlooping equiralent, denoted by $L_{1} \sim_{n} L_{2}$, if $L_{n}\left(L_{1}\right)=L_{n}\left(L_{2}\right)$ and are nonlooping inequivalent, denoted by $L_{1} \chi_{n} L_{2}$, if $\boldsymbol{L}_{n}\left(L_{1}\right) \neq \boldsymbol{L}_{n}\left(L_{2}\right)$. We also say that a language $L$ is nonlooping complete or $n$-complete if $\boldsymbol{L}_{n}(L)$ is the family of all nonlooping languages.

Theorem 3.1. The first density characterization theorem. Given two languages $L_{1}$ and $L_{2}$ with $L_{1}<L_{2}$, then $\left(L_{1}, L_{2}\right)$ is dense if and only if $L_{1} \sim_{n} L_{2}$. Similarly if $L_{1}$ and $L_{2}$ are regular, then $\left(L_{1}, L_{2}\right)$ is $r$-dense if and only if $L_{1} \sim_{n} L_{2}$.

Proof. The second statement follows from the first by way of Theorem 2.2, hence we will only prove the first statement here.

Without loss of generality assume both $L_{1}$ and $L_{2}$ are minimal.

If: Assume $L_{1} \sim_{n} L_{2}$. Observe that for all $L$ satisfying

$$
L_{1} \leqq L \leqq L_{2}
$$

we have $L \sim_{n} L_{i}, i=1,2$. Hence, if we show that for $L_{1} \sim_{n} L_{2}$ and $L_{1}<L_{2}$ there is an $L$ such that $L_{1}<L<L_{2}$, then the "if-part" follows immediately.

Let $L_{2}=L_{2}^{\prime} \cup M_{1} \cup \ldots \cup M_{m}$, for distinct, nontrivial coherent minimal nonlooping $M_{i}, 1 \leqq i \leqq m$ and $L_{2}^{\prime}$ looping, where $L_{2}^{\prime}$ cannot be further decomposed under $U$ into a nontrivial nonlooping language and a looping language. We say the above decomposition of $L_{2}$ is a maximal nonlooping decomposition of $L_{2}$. Similarly, let $L_{1}=L_{1}^{\prime} \cup K_{1} \cup \ldots \cup K_{k}$ be a maximal nonlooping decomposition of $L_{1}$. Note that $L_{1}^{\prime} \leqq L_{2}^{\prime}$, sinc a looping language cannot be an interpretation of a nonlooping one.

Since $L_{1} \sim_{n} L_{2}, M_{i} \leqq L_{1}, 1 \leqq i \leqq m$. Furthermore $M_{i} \neq L_{1}^{\prime}$ since if it vere, then $M_{i} \leqq L_{2}^{\prime}$ which contradicts the minimality of $L_{2}$. Therefore $M_{i} \leqq K_{j}$ for some $j$. Similarly $K_{j}$ is an interpretation of the same $M_{i}$, otherwise $L_{1}$ is not minimal. Hence 
$M_{i} \sim K_{j}$. This implies we can write $L_{1}$ as $L_{1}^{\prime} \cup M_{1} \cup \ldots \cup M_{m} \cup N_{1} \cup \ldots \cup N_{n}$, where $n \geqq 0$ and the $N_{i}$ are nontrivial, coherent, minimal, and nonlooping.

Note that $L_{2}^{\prime} \neq \emptyset$. Otherwise $L_{1}^{\prime}=\emptyset$ and $n=0$, hence $L_{1} \sim L_{2}$, a contradiction. Finally consider minimal $L_{3}$ and $L_{4}$ such that

$$
L_{1} \leqq L_{3}<L_{4} \leqq L_{2} \text {. }
$$

Then by similar arguments to those for $L_{1}$ above we can express $L_{3}$ as

and $L_{4}$ as

$$
L_{3}^{\prime} \cup M_{1} \cup \ldots \cup M_{m} \cup N_{1} \cup \ldots \cup N_{s}
$$

$$
L_{4}^{\prime} \cup M_{1} \cup \ldots \cup M_{m} \cup N_{1} \cup \ldots \cup N_{t} \text {, where } 1 \leqq t \leqq s \leqq n .
$$

Moreover $L_{3}^{\prime}$ can be expressed as $J_{1} \cup \ldots \cup J_{p}$ and $L_{4}^{\prime}$ as $K_{1} \cup \ldots \cup K_{q}$, where each of the $J_{j}$ and $K_{i}$ are looping and coherent. We now show that we can always construct an $L$ such that $L_{3}<L<L_{4}$, that is $\left(L_{1}, L_{2}\right)$ is dense.

(i) $s=t$. In this case there exists an $i$ such that for all $j, 1 \leqq j \leqq p$ either $J_{j} \neq K_{i}$ or $J_{j}<K_{i}$. For otherwise $L_{3}^{\prime} \sim L_{4}^{\prime}$ and hence $L_{3} \sim L_{4}$. Since $K_{i}$ is looping it has no predecessor (by Theorem 2.5). Therefore consider a $K_{i}^{\prime}<K_{i}$ which also satisfies $K_{i}^{\prime} \nsim J_{j}, 1 \leqq j \leqq p$. Such a $K_{i}^{\prime}$ must exist since there are only finitely many $J_{j} \leqq K_{i}$, but infinitely many inequivalent $K_{i}^{\prime}$ with $K_{i}^{\prime}<K_{i}$. To conclude this subcase observe that $L_{3} \cup K_{i}^{\prime}$ is properly between $L_{3}$ and $L_{4}$.

(ii) $s>t$. Now $N_{t+1} \cup \ldots \cup N_{s} \leqq K_{1} \cup \ldots \cup K_{q}$, otherwise $L_{3}$ would not be minimal. In particular this implies $N_{t+1} \leqq K_{i}$ for some $i, 1 \leqq i \leqq q$. Consider a $K_{i}^{\prime}$ such that $N_{t+1}<K_{i}^{\prime}<K_{i}$. Surely such a $K_{i}^{\prime}$ exists and furthermore as in subcase (i) $L_{3}<$ $L_{3} \cup K_{i}^{\prime}<L_{4}$.

Only if: Assume $\left(L_{1}, L_{2}\right)$ is dense. If $L_{1} \chi_{n} L_{2}$, then there exists a coherent nonlooping $N$ with $N \leqq L_{2}$ such that $N 末 L_{1}$. But this implies $L_{1}<L_{1} \cup N \leqq L_{2}$ and by Theorem 2.6 $L_{1} \cup P$ is a predecessor of $L_{1} \cup N$, if $P$ is a predecessor of $N$. But this implies $\left(L_{1}, L_{2}\right)$ is not dense, a contradiction.

Corolla ry 3.2. For an arbitrary regular language $L,\left(L, a^{*}\right)$ is $r$-dense if and only if $\boldsymbol{L}$ is $n$-complete and $\boldsymbol{L}_{\boldsymbol{r}}(L) \subset L(R E G)$.

Corollary 3.3. For two arbitrary languages $L_{1}$ and $L_{2}$ with $L_{1}<_{r} L_{2},\left(L_{1}, L_{2}\right)$ is not $r$-dense if $L_{1}$ is nonlooping.

This follows by observing that if $L_{2}$ is nonlooping then $L_{2} \neq L_{1}$ and hence $L_{1} \varkappa_{n} L_{2}$. On the other hand if $L_{2}$ is looping then it can generate arbitrarily long chains of words (or broken loops, see [MSW2]) and $L_{1}$ cannot. Hence once again $L_{1} \chi_{n} L_{2}$.

Corollary 3.4. The interval $\left(L, a^{*}\right)$ is not $r$-dense, where $L=\left(a^{*}-\left\{a^{2}\right\}\right) \cup$ $\{a b, b a, b\}$.

Proof. Consider the language $M=\{a b, a c d, b e f\}$. Clearly $M$ is nonlooping and $M$ is minimal and coherent. Now both $a$ and $b$ appear in a word of length 3 . Therefore 
letting $h$ be a morphism such that $h(M) \subseteq L$, it follows that $h(a c d)=h(b e f)=a a a$ and hence $h(a b)=a a$. But $a a$ is not in $L$, hence $M$ 丰 $L$ and by Corollary $3.2\left(L, a^{*}\right)$ is not dense.

To enable us to present specific $r$-dense intervals of the form $\left(L, a^{*}\right)$ we need to strengthen Theorem 3.1 for the case of $n$-completeness. This we now do by way of the following definitions.

Let $L \subseteq \Sigma^{*}$ be an arbitrary nonlooping language and let $L^{\prime}=L-\Sigma$. We say a word $w$ in $L$ is an end word if

$$
\operatorname{alph}(w) \cap \operatorname{alph}\left(L^{\prime}-\{w\}\right)=\{a\}, \text { for some } a \text { in } \Sigma .
$$

In this case we say a connects $w$ and $L^{\prime}-\{w\}$.

Lemma 3.5.

(i) Every nontrivial, coherent, nonlooping language $N$ has at least one end word if $\# N \geqq 2$.

(ii) If $N$ is a coherent, nonlooping language and $w$ is an end word in $N$, then $N-\{w\}$ is coherent.

Proof. Immediate.

We are now ready to state and prove our second characterization theorem.

Theorem 3.6. The second density characterization theorem. Let $L$ be an arbitrary language.

Then $\left(L, a^{*}\right)$ is $r$-dense if and only if $L$ has a nontrivial subset $L^{\prime}$ for which the following condition obtains:

For all letters $a$ in alph $\left(L^{\prime}\right)$ and for all $i, j \geqq 0$ there is a word $x$ in $\left(\operatorname{alph}\left(L^{\prime}\right)\right)^{i}$ and a word $y$ in $\left(\text { alph }\left(L^{\prime}\right)\right)^{j}$ such that xay is in $L^{\prime}$.

In other words $L$ is $n$-complete if and only if it has such a subset $L^{\prime}$.

Proof. In this proof whenever an $n$-complete language is mentioned we always assume it is also minimal in the sense that every proper subset of it is not $n$-complete. Clearly this is no loss of generality since each $n$-complete language has a minimal n-complete subset.

Because of Corollary 3.2 we only need consider the case that $L$ is $n$-complete, since $a^{*}$ is obviously $n$-complete. Moreover we observe that $L$ is $n$-complete if and only if $N \leqq L$ for every coherent nonlooping language $N$.

If: To show that $L$ is $n$-complete we need to prove that every nonlooping coherent language $N$ has a morphic image in $L^{\prime}$ and hence in $L$. We prove this by induction on the cardinality of $N$. Note that $L^{\prime}$ contains words of all lengths. For $\# N=1$, since the only word must consist of distinct letters it trivially has a morphic image in $L^{\prime}$.

Now assume that for some $k \geqq 1$, every coherent, nonlooping $N$ with $\# N \leqq k$, has a morphic image which is a subset of $L^{\prime}$. 
Let $N$ be a nonlooping language with \#N=k+1. For $w$ an end word in $N$ there is a morphism $h$ such that $h(N-\{w\})$ is a subset of $L$.

Consider the symbol $a$ which connects $w$ and $L$. Then we can write $w$ as $b_{1} \ldots$ $b_{i} a b_{i+1} \ldots b_{n}$, where $0 \leqq i \leqq n$. Clearly there is a word $v$ in $L^{\prime}$ satisfying

$$
v=x_{1} h(a) x_{2},
$$

where $\left|x_{1}\right|=i$ and $\left|x_{2}\right|=n-i$. Note that the letters $b_{1}, \ldots, b_{n}$ are distinct from each other and from alph $(N-\{w\})$. Hence we can extend $h$ to these new symbols such that $h(w)=v$. In other words $h(N) \subseteq L^{\prime}$ completing this part of the proof.

Only if: $L$ is minimal and $n$-complete by assumption, hence we prove it satisfies the property in the Theorem statement.

Let $a$ be a letter in alph $(L)$ and let $x a y$ be a word in $L$. Clearly there must be at least one such word otherwise $a$ would not be in alph $(L)$.

Now there is a nonlooping language $N$ such that whenever $h(N) \subseteq L$, then there is a word $w$ in $N$ with $h(w)=x a y$. If this is not the case $L-\{x a y\}$ is also $n$-complete, contradicting the minimality of $L$. We define nonlooping languages $M_{i j}$ for all $i, j \geqq 0$ by:

For every symbol $s$ in alph $(N)$ add a word

$$
a_{1} \ldots a_{i} s b_{1} \ldots b_{j}
$$

to $N$, where $a_{l}$ and $b_{m}$ are new symbols for every symbol $s$ in $\operatorname{alph}(N)$.

Now since each $M_{i j}$ is nonlooping we have $M_{i j} \leqq L$ for all $i, j \geqq 0$. Moreover whenever $g\left(M_{i j}\right) \subseteq L$, for some morphism $g$, then $g(w)=x a y$ by the above remarks Hence $g\left(a_{1} \ldots a_{i} s b_{1} \ldots b_{j}\right)=x_{1} a y_{1}$, for some $s$ in $\operatorname{alph}(N)$, where $\left|x_{1}\right|=i$ and $\left|y_{1}\right|=j$. Since $x_{1} a y_{1}$ is in $L, L$ satisfies the property in the 1 heorem statement, completing the proof.

This leads immediately to some specific examples of $n$-complete languages and hence dense intervals.

Corollary 3.7. $L_{1}=\{a, b\}^{*}-\left\{a^{i}, b^{i}: i \geqq 2\right\}$ is n-complete and hence $\left(L_{1}, a^{*}\right)$ is an $r$-dense interval.

Proof. $L_{1}$ clearly satisfies the condition of Theorem 3.6.

Corollary 3.8. $L_{2}=\{a, b, c\}^{*}-\left\{a^{3}, b^{3}, c^{3}, a a b, a a c, a b a, a c a, b a a, c a a, b b c, b c b\right.$, cbb\} is n-complete.

More importantly:

Corollary 3.9. Let $\Sigma_{m}=\left\{a_{1}, a_{2}, \ldots, a_{m}\right\}$ and $K_{m}=\left(\Sigma_{m}^{*}-\Sigma_{m}^{2}\right) \cup\left\{a_{1} a_{2}, a_{2} a_{3}, \ldots\right.$, $\left.a_{m} a_{1}\right\}$. Then $K_{m}$ is $n$-complete. 


\section{Decidability and maximality}

In this section we first prove that $n$-completeness is decidable for context-free languages, and then show that there is no maximally $r$-dense interval $\left(L, a^{*}\right)$.

The orem 4.1. $\mathrm{N}$-completeness is decidable for context-free languages.

Proof. $L$ is $n$-complete if and only if it has a subset $L^{\prime}$, which satisfies the condition of Theorem 3.6, that is $L^{\prime}=L \cap \Sigma^{*}$ for some $\Sigma \subseteq$ alph $(L)$. Now define finite substitutions $\delta_{a}$ for all $a$ in $\Sigma$ by:

$$
\begin{aligned}
& \delta_{a}(a)=\{f, a\} \\
& \delta_{a}(b)=\{f\}, \text { for all } b \text { in } \Sigma, b \neq a,
\end{aligned}
$$

where $f$ is a new symbol. Clearly $L^{\prime}$ satisfies the condition of Theorem 3.6. if and only if $M_{a}=\delta_{a}\left(L^{\prime}\right) \cap f^{*} a f^{*}$ equals $f^{*} a f^{*}$, for all $a$ in $\Sigma$.

This is decidable since $f^{*} a f^{*}$ is a bounded regular set and $M_{a}$ is context-free.

This together with Theorem 3.1 immediately gives:

Corollary 4.2. Given a context-free (regular) language $L$ it is decidable whether or not $\left(L, a^{*}\right)$ is dense ( $r$-dense).

In order to prove the maximality result we need to consider directed cycles of length $m$, denoted by $C_{m}$. Letting $\Sigma_{m}=\left\{a_{1}, a_{2}, \ldots, a_{m}\right\}$ we define $C_{m}$ by:

$$
C_{m}=\left\{a_{1} a_{2}, a_{2} a_{3}, \ldots, a_{m} a_{1}\right\} .
$$

It is a straightforward observation that

$$
C_{r} \leqq C_{m} \quad \text { if and only if } r=0(\bmod m) .
$$

On the other hand every nonlooping language $N \subset \Sigma^{2}$ is an interpretation of $C_{m}$ for all $m \geqq 1$.

We now have:

Lemma 4.3. Let $L$ be an $n$-complete language. Then there is an $m$ and a bijection $g$ such that $g\left(C_{m}\right) \subseteq L$.

Proof. We only need consider $L^{\prime}=\{w$ is in $L:|w|=2\}$. Let $\# L^{\prime}=r$. Now since all nonlooping languages are interpretations of $L$, then in particular

$$
P_{r}=\left\{a_{1} a_{2}, a_{2} a_{3}, \ldots, a_{r} a_{r+1}, a_{r+1} a_{r+2}\right\}
$$

where the $a_{i}$ 's are different letters for different $i$ 's, is an interpretation of $L^{\prime}$, that is there is a morphism $h$ such that $h\left(P_{r}\right) \subseteq L^{\prime}$. Now $h$ cannot be one-to-one, since \# $\boldsymbol{P}=r+1>\# L^{\prime}$. Therefore $h$ merges at least two letters and hence there is an $m \geqq 1$ such that $C_{m} \leqq h\left(P_{r}\right)$. But this implies $g\left(C_{m}\right) \leqq L^{\prime} \leqq L$ for some bijection $g$ completing the proof. 
We also need:

Lemma 4.4. Let $L_{1}$ and $L_{2}$ be (regular) languages. Then there is a (regular) language $L$ such that

and

$$
\boldsymbol{L}(L)=\boldsymbol{L}\left(L_{1}\right) \cap \boldsymbol{L}\left(L_{2}\right)
$$

$$
\boldsymbol{L}_{\boldsymbol{r}}(L)=\boldsymbol{L}_{\boldsymbol{r}}\left(L_{1}\right) \cap \boldsymbol{L}_{\boldsymbol{r}}\left(L_{2}\right) .
$$

Proof. This follows along the lines of the proof of Theorem 4.2 in [MSW5] and therefore it is left to the reader.

We are now able to prove our final result:

Theorem 4.5. There is no (regular) language $L$ such that $\left(L, a^{*}\right)$ is maximally dense ( $r$-dense).

Proof. We show that every dense interval $\left(L, a^{*}\right)$ can be extended. In other words that there exists an $L_{0}$ such that $L_{0}<L$ and $\left(L_{0}, a^{*}\right)$ is dense.

From Lemma 4.3 we know that there is an integer $m \geqq 1$ and a bijection $g$ such that $g\left(C_{m}\right) \subseteq L$. Let $m_{0}$ be the greatest such $m$.

Immediately $L^{\prime}=\{w$ is in $L:|w|=2\}$ is not an interpretation of $C_{m_{0}+1}$, since $C_{m_{0}} \neq C_{m_{0}+1}$.

Now consider $K_{m_{0}+1}$ from Corollary 3.9. Then $C_{m_{0}+1} \subseteq K_{m_{0}+1}$ and moreover is not an interpretation of $K_{m_{0}+1}$. Now let $L_{0}$ be a language such that

$$
\boldsymbol{L}\left(L_{0}\right)=\boldsymbol{L}(L) \cap \boldsymbol{L}\left(K_{m_{0}+1}\right) .
$$

Note that $L_{0}<L$, since $L$ is not in $L\left(K_{m_{0}+1}\right)$ and so it is not in $L\left(L_{0}\right)$.

It remains to demonstrate that $L_{0}$ is $n$-complete. However $L$ is $n$-complete by assumption and $K_{m_{0}+1}$ is $n$-complete by Corollary 3.9. Hence $L_{0}$ is $n$-complete and $\left(L_{0}, a^{*}\right)$ is both dense and an extension of $\left(L, a^{*}\right)$ as required.

If $L$ is regular, then $L_{0}$ can be chosen to be regular (Lemma 4.4) since $K_{m_{0}+1}$ is regular. Hence by Theorem 2.2, the "regular" version of the theorem follows.

\section{References}

[CG] Cremers, A. B., and S. Ginsburg: Context-free grammar forms. - J. Comput. System Sci. $11,1975,86-116$.

[GGS1] Ginsburg, S., J. Goldstine, and E. H. Spanier: A prime decomposition theorem for grammatical families. - J. Comput. System Sci. 24, 1982, 315-361.

[GGS2] Ginsburg, S., J. Goldstine, and E. H. Spanier: On the equality of grammatical families. J. Comput. System Sci. 26, 1983, 171-196.

[H] HaRrison, M. A.: Introduction to formal language theory. - Addison-Wesley Publishing Co., Inc., Reading, Mass., 1978.

[HU] Hopcroft, J. E., and J. D. Ullman: Formal languages and their relation to automata, Second edition. - Addison-Wesley Publishing Co., Inc., Reading, Mass., 1979. 
[MSW1] MaURer, H. A., A. SalomaA, and D. Wood: Colorings and interpretations: A connection between graphs and grammar forms. - Discrete Appl.. Math. 9, 1981, 119-135.

[MSW2] MAUReR, H. A., A. SAlOMAA, and D. Wood: On predecessors of finite languages. - Inform. and Control 50, 1981, 259-275.

[MSW3] Maurer, H. A., A. SalomaA, and D. Wood: Dense hierarchies of grammatical families.J. Assoc. Comput. Mach. 29, 1982, 118-126.

[MSW4] Maurer, H. A., A. SalomaA, and D. Wood: Finitary and infinitary interpretations of languages. - Math. Systems Theory 15, 1982, 251-265.

[MSW5] Maurer, H. A., A. SalomaA, and D. Wood: On finite grammar forms. - Internat. J. Comput. Math. 12, 1983, 227-240.

[N] Niemi, V.: Personal communication, 1984.

[OSW] Ottmann, Th., A. SalomaA, and D. Wood: Sub-regular grammar forms. - Inform. Process. Lett. 12, 1981, 184-187.

[S] SalomaA, A.: Jewels of formal language theory. - Computer Science Press, Inc., Rockville, Maryland, 1981.

[Wo] Wood, D.: Grammar and L forms; An introduction. - Lecture Notes in Computer Science 91, Springer-Verlag, New York, 1980.

TU Graz

Institute für Informationsverarbeitung

Schießstattgasse $4 \mathrm{a}$

A-8010 Graz

Austria

TU Graz

Institute für Informationsverarbeitung

Schießstattgasse $4 a$

A-8010 Graz

Austria

Received 11 October 1984
University of Turku

Department of Mathematics

SF-20500 Turku

Finland

University of Waterloo

Department of Computer Science

Waterloo, Ontario N2L 3G1

Canada 